\title{
Routing and Wavelength Assignment in GMPLS-based 10 Gb/s Ethernet Long Haul Optical Networks with and without Linear Dispersion Constraints
}

\author{
Le Nguyen BINH ${ }^{1,2}$ \\ ${ }^{I}$ Department of Electrical and Computer Systems Engineering, Monash University, Australia \\ ${ }^{2}$ Lehrstuhl für Nachrichen- und Übertragungstechnik, \\ Technische Fakultaet der Christian Albretchs Universitaet zu Kiel, Germany \\ E-mail:le.nguyen.binh@eng.monash.edu.au
}

\begin{abstract}
Given a set of lightpath connection requests in an all-10 Gb/s optical dense wavelength division multiplexed (DWDM) Ethernet network, lightpaths are designed. In addition the wavelength channels are assigned subject to minimization of the channel blocking and provisional requests satisfying the limits due to accumulative linear dispersion effects over the hops.

This paper proposes a routing and wavelength assignment scheme for DWDM long-haul optical networks that includes routing, assignment and reservation of different wavelength channels operating under the Generalized Multiprotocol Label Switching (GMPLS) environment. The GMPLS framework can offer an approach to implement IP over DWDM with variable weighting assignments of routes based on the limitations due to residual dispersion accumulated on the lightwave path.

The modeling is implemented under the framework of an object-oriented modeling platform OMNeT++. Network performance tests are evaluated based mainly on a long-haul terrestrial fiber mesh network composed of as well as three topologies structured as chain, ring, and mesh configurations. Blocking probability of lightpath connection requests are examined with the average link utilization in the network employing variable number of wavelength channels in association with the limits of route distance due to linear chromatic and polarization mode dispersion effects.
\end{abstract}

Keywords: DWDM Optical Networks, Optical Transmission Systems, GMPLS, Routing and Wavelength Assignment (RWA), Wavelength Routers.

\section{Introduction}

In recent years, since the invention of optical amplifiers in late 1980s advances in dense wavelength division multiplexing (DWDM) technology has enabled the implementation of multi-Tera/bits/sec. capacity in intelligent optical networks (ION) [1]. In particular 10 $\mathrm{Gb} / \mathrm{s}$ Ethernet optical networks over DWDM have been standardized and emerges as the most likely ultra-fast information networks in the near future. Undoubtedly systems and networks need to be interconnected in the optical wavelength domain or layer via optical routers and photonic switches and managed under appropriate protocols. The DWDM technology offers the capability of building very large wide area network (WAN) consisting of thousands of nodes with per-node throughputs of the order of tens of $\mathrm{Gb} / \mathrm{s}$.

However, at $10 \mathrm{~Gb} / \mathrm{s}$ and possibly higher with the possibility of upgrading certain number of channels to $40 \mathrm{~Gb} / \mathrm{s}$ or even $100 \mathrm{~Gb} / \mathrm{s}$, the detrimental effects of linear dispersion effects such as the chromatic dispersion (CD) and polarization mode dispersion (PMD) and are critical for error free transmission and interconnect between the IP routers. Furthermore, the number of wavelength channels are high with the average total power may be over the nonlinear threshold for the transmission lightpaths that would lead to the reduction of the eye opening of the receiver signals. We must note that these effects are not very critical for $2.5 \mathrm{~Gb} / \mathrm{s}$ transmission bit rate which is now commonly used in 
present optical networks.

In this paper we include only the residual linear dispersion and the PMD effects into the investigation of traffic engineering in IP over WDM optical networks, especially the routing and wavelength assignment (RWA) of wavelength channels over fiber paths of the networks. We understand that these transmission issues have not ever been taken into account in published research works. Due to the linear dispersion tolerance and nonlinear effects currently several advanced modulation formats have been studied [1] in order to combat these impairments. Usually these distortion effects are dependent on the length of the transmission that is commonly faced in all optical networks, especially in backbone WDM networks.

Routing and wavelength assignment (RWA), i.e. a cross-connecting of several wavelength allocated channels between DWDM optical networks, is very important [3]. One unique feature of DWDM networks is the tight coupling between routing and wavelength selection. Routing and wavelength assignment (RWA) problem is a major and complex problem associated with optical networks. Thus, given that (i) a set of dynamic and randomly chosen lightpaths that need to be established; (ii) constraints of the total number of wavelengths propagating in an optical fiber; and (iii) wavelength continuity constraint (WCC), which means a lightpath must use the same wavelength on all the links along its path from source to destination edge node, The controller for routing and assignment of wavelength channels must determine the routes over which these lightpaths should be set up and determine the wavelengths to be assigned to these lightpaths so that an optimum number of lightpaths may be established for the routing. Each wavelength channel is associated with a dispersion factor. Due to the dispersion slope of the transmission fiber, the dispersion compensation using dispersion compensating fiber is normally fully compensated at the center wavelength of the wavelength band. Thus there exists residual chromatic dispersion (CD) at other channels. At $10 \mathrm{~Gb} / \mathrm{s}$ these residual dispersion effects are very critical. Furthermore the dispersion due to different traveling velocity of polarized modes of the single mode fiber, the polarization mode dispersion (PMD) is also important and must be taken into account.

Lightpaths that cannot be set up due to constraints on routes or wavelengths or the linear CD and PMD at which the eye opening penalty (EOP) at the receive end would suffer $3 \mathrm{~dB}$, are said to be blocked, so the corresponding network optimization problem is to maximize the probability of set-up for a current connection request while minimizing the blocking probability of future connection requests. Furthermore in long haul terrestrial and or intercontinental optical networks, a generic management of the whole network status is preferred.

The concept of GMPLS offers an approach to implement IP over DWDM [4-10]. Indeed, GMPLS becomes evident that it is the best control plane solution for next-generation optical networking. In GMPLS, the MPLS label is generalized so that a label can also be encoded as a time slot, a wavelength, or a spatial identifier. The embrace by GMPLS is extremely large; the most critical part of it is to solve the general problem of dynamic lightpath establishment.

Therefore, in this paper various schemes for RWA algorithms are studied, compared and selectively implemented on a long haul optical terrestrial mesh network based on a common control plane GMPLS framework, especially when residual chromatic dispersion and polarization mode dispersion effects are taken into account. The nonlinear phase noises and impairments in network routing and wavelength assignment will be reported in a future article. Other network topologies also are investigated in this work such as a 4-node/8-node chain, a 5-node ring, and a terrestrial mesh network. We studied two kinds of wavelength assignment methods in all-optical networks: First-fit (Fixed-order) and random wavelength assignment, and two kinds of link metrics: simple total and available wavelength (TAW) and enhanced TAW. We use OMNeT++ [12] to simulate GMPLS-based alloptical networks and compare the blocking probabilities of these RWA methods on different topologies and traffic models. The simulation results show that first-fit wavelength assignment has a lower blocking probability than random wavelength assignment, and enhanced TAW performs better than simple TAW when link utilization becomes high. We also found that under some circumstances the difference in blocking probability may be widened and worsened due to additional limitations due the total accumulated dispersion along a routed lightpath.

The paper is organized as follows: Section 2 outlines the physical constraints on the routings of wavelength channels over long haul optical networks whose parameters are then declared. Also an ultra-fast automatic wavelength router is proposed. The specific characteristics of GMPLS are given. Section 3 and 4 propose the RWA algorithm and structures of simulation platform. The issues involved RWA problems in such linear dispersion (CD and PMD) effects are given. Our simulation model and simulation results are given in Section 5. Traffic performances of the proposed RWA under different network scenario under with and without dispersion effects are obtained and described. Finally, conclusion and further works are found in Section 6.

\section{Network Physical Constraints}

Routing and wavelength selection are coupled tightly in 
WDM all-optical networks. When establishing a connection in optical networks, both routing (path selection) and wavelength assignment (allocating a wavelength along the selected path) must be considered. This is referred to as routing and wavelength assignment (RWA) problem. This problem is more complex than routing problem in electronic networks. Optical networks operating at lower bit rates less than $2.5 \mathrm{~Gb} / \mathrm{s}$ can be routed under constraints of [3]: (i) Wavelength continuity constraint (WCC): a lightpath must use the same wavelength on all the links along its path from source to destination edge node; (ii) Distinct wavelength constraint: all lightpaths using the same link (fiber) must be allocated distinct wavelengths; (iii) Limited number of wavelength per fiber link is restricted within the Cband and by the nonlinear threshold, mainly due to the self phase modulation (SPM) effects. Furthermore for higher bit rates $10 \mathrm{~Gb} / \mathrm{s}$ and above additional constraints are (iv) Total fiber routing path is limited due to the total residual dispersion allowable on the total routing distance that suffer an eye opening penalty of $3 \mathrm{~dB}$; and (v) Total accumulated noise and hence EOP due to amplified stimulated amplification within optical amplifiers placed in each fiber spans that is important for backbone long haul networks. These noise contributions are ignored in this work.

It is noted here that the dispersion compensation can normally be made for a specific wavelength of the Cband and there are always residual dispersion mismatched at other wavelength channels due to dispersion slopes. Thus the effects of residual dispersion are very critical in the design of the DWDM network routing and wavelength assignment as these dispersion effects are length dependent.

\subsection{Wavelength Continuity Constraint (WCC)}

The WCC is a unique constraint of WDM all-optical networks. In WDM networks, if a connection is accepted, it should be assigned a path through the network and a wavelength. This wavelength must be the same wavelength on all links along the path. Consequently, it is likely that any algorithm engaging WCC would suffer higher blocking probability. If another lightpath has to be set up between node 1 and node 3 , it will be blocked, because there is no available wavelength along its lightpath (i.e. no common available wavelength on both links). Due to high costs of all-optical wavelengthconversion photonic components, it is assumed that none of the optical cross connectors (OXCs) has wavelength conversion capability.

The algorithm must establish dynamically an end-toend path between any ingress nodes and any egress nodes in the all-optical network. Our main goal is to minimize the blocking of lightpaths in the network. Different metrics for dynamic routing algorithms are examined. For those parameters which are not wavelength-based then constraints are not taken into account, unlike in other published works physical constraints are integrated them in the routing decision process [1].

\subsection{Distinct Wavelength Constraint}

Different lightpaths on the same physical link (fiber) must use different wavelengths. Each fiber in WDM networks may contain several WDM wavelengths subject to power below specific power threshold due to nonlinear effects. However these nonlinear effects are ignored in this report. It is assumed that the operating wavelengths are placed in a 50,100 or $200 \mathrm{GHz}$ grid $(0.4 \mathrm{~nm}$ wavelength spacing) with a nominal center frequency of $193.1 \mathrm{THz}(1552.52 \mathrm{~nm})$ in the middle of the $1.55 \mu \mathrm{m}$ fiber and EDFA pass-band [4]. More details are specified in ITU G.692.

\subsection{Number of Wavelengths per Fiber Link}

Consider one link between two adjacent nodes. Each link cable may be composed of several fibers in which several multiplexed wavelength channels are transmitted. Figure 1 shows a typical automatic wavelength optical cross connect (OXC) including wavelength converters, wavelength switching matrix whose control signals come from the central management systems complying the state of the designed algorithm and global information obtained from the nodes of the networks. The wavelength converter is a semiconductor optical amplifier biased to operate for wavelength conversion. This conversion is associated with the wavelength multiplexer so that once the conversion is completed to a wavelength output port the channel is routed immediately to the output. Thus this structure allows an ultra-fast routing. The limit of the operation of this OXC depends on the conversion speed of the SOA which is, under present technology, in order of picoseconds for $\mathrm{GHz}$ operation.

Let $\lambda_{i, j}$ be the wavelength number $i$ in the fiber $j$. Assuming the operating wavelength range is the C-band. The wavelengths range in the C-band is $1,530 \mathrm{~nm}$ to $1,565 \mathrm{~nm}$. Each wavelength is assigned a specific emission wavelength that is specified by the ITU-T. Consequently, it is assumed that there is a maximum of 44 wavelengths per fiber with $100 \mathrm{GHz}$ spacing between channels. The wavelengths are ordered as follows: $\lambda_{1, j}=$ $1,530.33 \mathrm{~nm}$ and $\lambda_{44, \mathrm{j}}=1,564.68 \mathrm{~nm}$, with $\mathrm{j}$ being the number of fibers used. In a nutshell, the physical-based wavelength constraints are:

$$
\left\{\begin{array}{l}
\lambda_{i, j}=\text { wavelength } i \text { in } j^{\text {th }} \text { fiber.........i } \in 1 \div 44 \\
\lambda_{1, j}=1,530.33 \mathrm{~nm} ; \lambda_{44, j}=1,564.68 \mathrm{~nm}(C-\text { band })
\end{array}\right.
$$


wavelength channels propagating in the same fiber path.

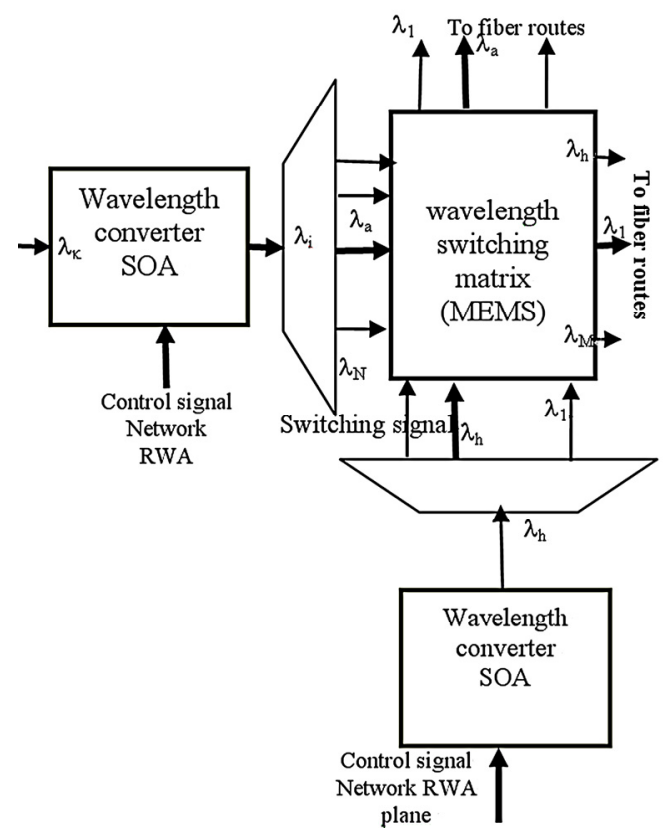

Figure 1. Schematic of an optical cross connect (OXC) incorporating wavelength conversion and routing by an SOA and demultiplexer and switching matrix for routing a specific wavelength to a designated output port for further routing.

Still, it can be shown that a multi-fibre RWA problem is algorithmically equivalent to a single fibre based optical network. Thus, in the following, an ordered list of wavelengths with only one fibre is considered. Further it is very unlikely that the 44 wavelengths transmitted or available in the $\mathrm{C}$ band will be all practically employed. Usually only a few wavelength channels are lit. Thus it is reasonable to assume that the population of the WDM slots is limited to a multiple of 8 wavelengths, up to 32 or 40 with $100 \mathrm{GHz}$ spacing between wavelength channels. As the number of fibers and the optical power launched in each wavelength, there are possibilities that the total average power propagating through a fiber path would reach above the nonlinear threshold (normally $3 \mathrm{dBm}$ ) and hence nonlinear phase distortion. In this work we set the limit at $10 \mathrm{dBm}$ taking into account the randomness of the chance of simultaneous transmission of all channels.

\subsection{Residual Linear Dispersion Constraints}

For high capacity long-haul transmission employing external modulation, the dispersion limit can be estimated in the following equation $[38,39]$.

$$
L_{D}=\frac{10^{5}}{D \cdot B_{R}{ }^{2}}
$$

where $\mathrm{D}$ is the dispersion factor and $\mathrm{B}_{\mathrm{R}}$ is the bit rate, $L_{D}$ is the dispersion length.

\subsection{Polarization Mode Dispersion Limit}

The length limit by the PMD effect can be defined as follows. Constraint on the dispersion length due to PMD must be included and an estimate of the transmission length limit due to PMD effect is given as [39]

$$
L_{P M D}=\frac{0.02}{\langle\Delta \tau\rangle^{2} \cdot B_{R}^{2}}
$$

with $L_{P M D}$ is the maximum length due to the PMD effect that generates a $1 \mathrm{~dB}$ penalty on the eye opening.

\subsection{GMPLS Framework}

Generalized Multiprotocol Label Switching (GMPLS) extends the label switching architecture proposed in MPLS to other types of non-packet based networks, such as SONET/SDH based networks and WDM networks. It has recently become evident that GMPLS is the unified control plane solution for ultra-high capacity, ultra-fast optical networking. In GMPLS, the MPLS label is generalized so that a label can also be encoded as a time slot, a wavelength, or a spatial identifier. The basis of the GMPLS framework is defined in [5,9], including two main parts of routing and signaling.

GMPLS routing proposes extensions to interior gateway protocols (IGP) of routing being the open shortest path first extended for traffic engineering (OSPF-TE) [7] and intersystem to intersystem extended for traffic engineering (ISIS-TE) [8]. The protocols provide the distribution of link state information with traffic engineering information and attributes such as network topology, resource availability, and administrative constraints.

GMPLS signaling protocols include reservation protocol extended for traffic engineering (RSVP-TE) [10] and the constrained label distribution protocol (CR-LDP) of the MPLS-TE framework [11]. The GMPLS signaling protocols extend certain base functions of the RSVP-TE and CR-LDP signaling, which originally configure and control the distributed label switched paths (LSP). The extensions impact basically on LSP properties on how labels are requested and communicated, unidirectional nature of LSPs, how errors are propagated, and information provided for synchronizing the ingress and egress nodes.

\section{Routing and Wavelength Assignment}

RWA algorithm must be able to identify the maximum of explicit routes in IONs in order to satisfy dynamic routing requests. The RWA algorithm separates routing, wavelength assignment (WA) and reservation 
mechanisms. The search of an explicit route in the network that would meet the physical constraints of the ION is the principal aspect. Such a separation between routing and reservation protocols eases further works.

RWA algorithm is designed so that it gives an output an explicit route in the ION that determines each node to be traversed by the lightpath. Besides, a WA algorithm allows the determination of a wavelength channel satisfying the WCC that is reserved on the lightpath. Explicit routes and their associate wavelengths are then treated as the inputs of a reservation protocol in order to establish the optical circuit in the network. Present work develops a RWA scheme model of a GMPLS-based ION based on an adaptive link-state routing using global network knowledge including the rise time and dispersion budget of proposed routing link/distance. This choice is due to the followings. Table 1 shows a summary of the WA algorithms on a RWA problem.

Table 1. WA (wavelength assignment) algorithms in the RWA problem

\begin{tabular}{|c|c|c|c|}
\hline $\begin{array}{l}\text { WA } \\
\text { heuristics }\end{array}$ & Characteristics & Advantage & Drawback \\
\hline First-fit & $\begin{array}{l}\text { The wavelengths are } \\
\text { indexed, and a } \\
\text { lightpath will attempt } \\
\text { to select the } \\
\text { wavelength with the } \\
\text { lowest index before } \\
\text { attempting to select a } \\
\text { wavelength with a } \\
\text { higher index }\end{array}$ & $\begin{array}{l}\text { If global } \\
\text { information, } \\
\text { outperforms } \\
\text { random } \\
\text { heuristics. }\end{array}$ & $\begin{array}{l}\text { Possible } \\
\text { blocking if } \\
\text { simultaneous } \\
\text { lightpath } \\
\text { connections. }\end{array}$ \\
\hline Random & $\begin{array}{l}\text { Select one of the } \\
\text { wavelengths at } \\
\text { random }\end{array}$ & $\begin{array}{l}\text { If local } \\
\text { information, } \\
\text { outperforms } \\
\text { first-fit } \\
\text { heuristics. } \\
\text { Very simple. }\end{array}$ & $\begin{array}{l}\text { Does not } \\
\text { provide } \\
\text { optimal } \\
\text { wavelength } \\
\text { assignment. }\end{array}$ \\
\hline Least-used & $\begin{array}{l}\text { The wavelength } \\
\text { which is the most } \\
\text { used in the rest of the } \\
\text { network is selected. }\end{array}$ & $\begin{array}{l}\text { Spreads the } \\
\text { load evenly } \\
\text { across all } \\
\text { wavelengths }\end{array}$ & $\begin{array}{l}\text { Global } \\
\text { information } \\
\text { needed. }\end{array}$ \\
\hline Most-used & $\begin{array}{l}\text { The wavelength } \\
\text { which is the most } \\
\text { used in the rest of the } \\
\text { network is selected. }\end{array}$ & $\begin{array}{l}\text { Provides } \\
\text { maximum } \\
\text { wavelength } \\
\text { reuse in the } \\
\text { network }\end{array}$ & $\begin{array}{l}\text { Global } \\
\text { information } \\
\text { needed. }\end{array}$ \\
\hline
\end{tabular}

\subsection{Routing}

Several types of RWA algorithms have been studied. Three main types of routing schemes are fixed, fixedalternate and dynamic routing. Fixed and fixed-alternate routing principles are very simple. However, they have poor capabilities when dealing with self-healing capabilities and can yield a very high blocking probability under dynamic network operations. Thus, dynamic routing is considered.

The main disadvantage of dynamic routing is possibility for high overhead due to route advertisements. Nevertheless, the choice of an algorithm is always a trade-off between network performance and traffic overhead. Considering earlier research studies presented in $[3,12,13]$, it has been thought that a dynamic routing is one of the best ways to solve the RWA problem.

In the first instance, both adaptive routing schemes, that is link-state routing and distance-vector routing are considered. Contrary to link-state based routing algorithms that flood packets onto the network distancevector algorithms send their route advertisements only to their neighbors. In link-state algorithms, the link state update is flooded onto the whole network or area of OSPF.

\subsubsection{Distance-vector Routing Algorithm}

A distance-vector routing approach needs to use a distributed algorithm such as the popular Bellman-Ford algorithm. It is possible that a distance-vector algorithm can minimize the traffic overhead in the network while still giving relatively low blocking probabilities under high loads. A distance-vector algorithm is also interesting when considering constraint routing. Indeed, it is a property of the Bellman-Ford algorithm that, at its $h$-th iteration, it identifies the optimal (in our context: maximal number of wavelengths) path between the source and each destination, among paths of at most $h$ hops. This is recommended for QoS routing implementations [14]. Furthermore the distance vector is also tagged with the dispersion and distortion estimated for the routing distance.

Bellman-Ford algorithm progresses by increasing hop count, it essentially provides for free the hop count of a path as a second optimization criterion. This property is very interesting when applied to all-optical networks. However, even if the shortest path is sacrificed for a longer - in hops - route, the lightpath should not be too long as this could lead to a poor optical power and dispersion budgets.

Routing Information Protocol (RIP) is the most popular implementation of a distance-vector based protocol because it is simple and it is well suited to small networks. However, RIP has several flaws that make it unsuitable for ION. Particularly, RIP is unsuitable for large configurations and the convergence of the algorithm can also be lengthy; it suffers also from the count-to-infinity problem, of which the best remedy is to implement link-state algorithms.

\subsubsection{Link-state Based Routing Algorithm}

In link-state based routing, information is only sent when changes occur. A node builds up first a description of the topology of the network. Then it may use any routing algorithm to determine the route.

In the context of the ION, in order to compute an explicit route, it is also much easier to use a link-state based routing algorithm. Indeed, the lightpath to be established is more optimal if each node has a global knowledge of the network. It is also a property of the 
Dijkstra algorithm that a complete route from a source to any other node in the network can be easily found by recursive iteration on the graph.

Link-state based algorithms can use any routing algorithm. Different RWA algorithms can be implemented based on different optimization schemes (partial or total wavelength knowledge).

The open shortest path first (OSPF) protocol, known mostly for its second version [23], is the most widely known link-state based routing protocol and is employed for the simulation platform of this work. It has been increasingly popular over RIP, because it is most suitable for large networks. OSPF is an open source algorithm that can be found in different languages, including $\mathrm{C}++$. This is a particular advantage for simulation program based on $\mathrm{OMNeT}++$, a $\mathrm{C}++$ based simulator.

\subsubsection{Route Advertisements}

The route advertisement messages are built according to the OSPF extensions for GMPLS. This work proposes to extend the sub type length value (TLV) field relative to the interface switching capacity descriptor (ISCD) field of the GMPLS OSPF extensions.

In order to dynamically monitor the state of the network, each GMPLS router keeps track of all the wavelength capabilities of the whole network in a links database. This database is constantly updated each time a "routing" message is received. The "routing" message contains the description of the wavelength capabilities of a certain link that have changed very recently.

Those "routing" messages are actually flooded on the whole network by the node which one of its links' capabilities changed containing the address of the extremities of the link of which the wavelength capability has changed and the state of the changed wavelength.

The links database is composed of records, where each record describes one link of the network. Each record contains the node addresses of the link's extremities, a wavelength capability field and a metric field. The wavelength capability field describes explicitly the wavelength resources of the considered link. The metric field is the cost to use this link when performing the shortest path calculation. In this work, two different metrics have been implemented. They are both function of the total number of wavelengths and the number of available wavelength(s) on the link as described in the next part.

\subsubsection{Link Metrics}

The link metrics represent the costs to use certain links in the network. Intuitively, the link cost is a linear function of the total number of wavelength and the number of available wavelengths and the residual dispersion if taken into account.

The routing scheme is tested under different link metrics: simple total and available wavelength (TAW) or enhanced TAW. Let $\lambda_{i, j}^{a}$ be the number of available (unused) wavelengths on the link $(i, j)$ and the total number of possible wavelengths on that link. The simple TAW metric represents the load assigned to a link and is defined by:

$$
w_{i, j}=1-\frac{\lambda_{i, j}^{a}}{\lambda_{i, j}^{T}}, \forall(i, j) \in E
$$

The enhanced TAW metric is to test the metric proposed by [24] that equivalently minimizes the probability of blocking on an explicit route in which the weights are assigned following a log scale as:

$$
w_{i, j}=-\log \left[1-\left(1-\frac{\lambda_{i, j}^{a}}{\lambda_{i, j}^{T}}\right)^{\lambda_{i, j}^{a}}\right], \forall(i, j) \in E
$$

If residual dispersion is accounted then a constant or a linear or quadratic function of dispersion factor can be added into (4) or (5).

\subsubsection{Path Calculation}

The links database that has been freshly updated by the GMPLS router serves as the basis of the path discovery calculation based on the Dijkstra algorithm. This path computation is performed by each node in the network when it receives a "routing" message, which is an update of a certain link capability in the network.

The routing algorithm actually allows each node to build its own photonic database that contains $\mathrm{N}$ records, where $N$ is the number of nodes of the network. Each record is based on the following structure: (i) a node destination address - this identifies the record; it is the node to reach; (ii) a total cost to this destination node this is the total cost when taking the shortest path route to the destination node; (iii) an address of the last-butone node on the shortest path route to the destination node; (iv) an end-to-end available wavelength capability, which determinates explicitly the possible wavelengths to be assigned, if any, on the shortest path route; and (v) an explicit route holding an ordered list of all the addresses of the nodes on the shortest path route.

Given the links database, the first three fields are actually the direct output of the Dijkstra shortest path algorithm [14]. The last two fields are the result of a sub-routine of the Dijkstra algorithm. Indeed, it is a property of the Dijkstra algorithm that it is possible to find the list of the nodes of each shortest path by a simple recursive call.

We have also set constraints on the dispersion and attenuation of the fiber paths, in particular the polarization mode dispersion factor (PMD) as PMD is 
unlike the chromatic dispersion dependent on the fiber length. For chromatic dispersion we assume that the (standard single mode fiber) SSMF is compensated with dispersion compensating fiber and only residual dispersion is used. At $10 \mathrm{~Gb} / \mathrm{s}$ these dispersion effects are very critical. Additional nonlinear phase and hence distortion effects are not considered in this work.

\subsubsection{Lightpath Establishment}

There are two categories of lightpath establishment, one is the static lightpath establishment (SLE), and the other is dynamic lightpath establishment (DLE).

\subsubsection{Static Lightpath Establishment}

Static lightpath establishment (SLE) is used in the design and capacity planning phase of architecting an optical network. It can be logically decomposed into four subproblems. Assuming no wavelength conversion, the subproblems are listed as follows [33]: (i) Topology subproblem: determine the logical topology to be imposed on the physical topology, that is, determine the lightpaths in terms of their source and destination edge nodes; (ii) Lightpath routing sub-problem: determine the physical links which each lightpath consists of, that is, route the lightpaths over the physical topology; (iii) Wavelength assignment sub-problem: Determine the wavelength each lightpath uses, that is, assign a wavelength to each lightpath in the logical topology so that wavelength restrictions are obeyed for each physical link; and (iv) Traffic routing sub-problem: Route packet traffic between source and destination edge nodes over the logical topology obtained.

The SLE is also referred as static RWA problem, can be formulated as an integer linear program (ILP) where the objective is to maximize the number of connections that are successfully routed. A number of studies have investigated the static RWA problem.[12,15,17,29]. The ILP formulations are NP-complete and therefore may only be solved for very small systems. For large systems, heuristic methods must be used. A number of heuristics were proposed for the problem, they can be divided in four classes [17]: (i) heuristic solutions of the mixed integer linear programming problem; (ii) maximizations of the single-hop traffic flows; (iii) heuristic maximizations of the single-hop and multi-hop traffic flows; (iv) algorithms based on the adoption of a preestablished regular logical topology and on the optimization of the nodes placement according to the traffic pattern.

\subsubsection{Routing Sub-problems}

Fixed Routing - The most straightforward approach to routing a connection is to always choose the same fixed route for a given source-destination pair. This is called fixed routing. Fixed routing approach is easy to implement. However, it is very limited in terms of routing options. If resources (wavelengths) along the path are tied up, it can potentially lead to high blocking probabilities in the dynamic case, or may result in a large number of wavelengths being used in the static case. Moreover, fixed routing may be unable to handle fault situations in which one or more links in the network fail.

Fixed-Alternate Routing - An approach to routing that considers multiple routes is fixed-alternate routing. It increases the likelihood of establishing a connection by taking into account network state information. In fixed-alternate routing, each node in the network is required to maintain a routing table that contains an ordered list of a number of fixed routes to each destination node. Fixed-alternate routing provides simplicity of control for setting up and tearing down lightpaths and it may also be used to provide some degree of fault tolerance upon link failures. It can significantly reduce the connection blocking probability compared to fixed routing.

Adaptive Routing - In adaptive routing, route form a source node to a destination node is chosen dynamically, depending on the network state. The network state is determined by the status of all connections that are currently in progress. In order to choose an optimal route, a cost is assigned to each link in the network based on current network state information, such as wavelength availability on links, the total residual dispersion amount. A least-cost algorithm is then executed to find the minimum-cost route. Whenever a connection is established or taken down, the network state information is then updated.

\subsection{Wavelength Assignment}

In general, if there are multiple feasible wavelengths between a source node and a destination node, then a wavelength assignment algorithm is required to select a wavelength for a given lightpath. The wavelength selection may be performed either after a route has been determined, or in parallel with finding a route.

Since the same wavelength must be used on all links in a lightpath (noted that wavelength conversion is out of scope of this paper), it is important that wavelengths are chosen in a way which attempts to reduce blocking for subsequent connections. For the case that there are multiple feasible wavelengths between a source node and a destination node, heuristic methods must be used to assign wavelengths to the lightpath. A review of wavelength-assignment approaches can be found in [13] as follows: (i) Random: this scheme first searches the space of wavelengths to determine the set of all wavelengths that are available on the required route, randomly choose one wavelength (usually with uniform probability). (ii) First-Fit or fixed-order, in which all wavelengths are numbered. After searching for the 
available wavelengths, a wavelength with lowest index is chosen. The idea behind this scheme is to pack all of the in-use wavelengths toward the lower end of the wavelength space so that more wavelengths with higher indexes can be used for longer-hop connections. (iii) Least-Used selects the available wavelength that is the least used in the network, so the load is balanced on all the wavelengths. (iv) Most-Used is the opposite scheme of Least-Used, it always choose the available wavelength that is most used in the network. It packs connections into fewer wavelengths. (v) Min-Product is used in multi-fiber networks. In single-fiber networks, it is the same as First-Fit. It packs wavelengths into fibers, minimizes the number of fibers in the networks. (vi) Least-Loaded is also used in multi-fiber networks. It chooses the wavelength that has the largest residual capacity on the most-loaded link along route $p$. (vii) Max-Sum is proposed for multi-fiber networks but can also be used in single-fiber case. Generally speaking, the scheme considers all possible paths (lightpaths with their pre-selected routers) in the network and attempts to maximize the remaining path capacities after lightpath establishment. (viii) Relative Capacity Loss (RCL) is based on Max-Sum. RCL calculates a Relative Capacity Loss for each path on each available wavelength and then chooses the wavelength that minimizes the sum of the relative capacity loss on all the paths [36]. (ix) Wavelength Reservation reserves some wavelengths for multi-hop connections. This scheme reduces the blocking for multi-hop traffic but sometimes may overpunish single-hop traffic; and (x) Protecting Threshold, in which a single-hop connection is assigned a wavelength only if the number of idle wavelengths on the link is at or above a give threshold.

In this work the first-fit and random schemes are also selected for modeling the lightpath setup and RWA schemes in GMPLS-based IP-over-DWDM optical network. First-fit chooses the first wavelength available in an ordered list of wavelength in contrast to the random scheme which chooses a wavelength randomly between the different available wavelengths. Those two schemes are used directly at the output of the path calculation algorithm in order to determine the wavelength to be reserved on the path to each destination in the network from a source node.

\subsection{Signaling and Resource Reservation}

In order to set up a lightpath, a signaling protocol is required to exchange control information among nodes and to reserve resources along the path. In most cases, the signaling protocol is closely integrated with the RWA algorithms.

Signaling and resource reservation protocols may be categorized based on whether the resources are reserved on each link in parallel, reserved on a hop-by-hop basis along the forward path, or reverse path (backward). Algorithms will also differ depending on whether global information including the dispersion factors of each hop is available or not.

\section{Model Implementation}

\subsection{OMNeT++ Simulation Model}

$\mathrm{OMNeT}++$ is a free discrete event network simulation tool, which was primarily designed for the simulation of communication networks, multi-processors and other distributed systems [5]. The OMNeT++ based model is built to test two link metrics mentioned previously: the simple TAW and the enhanced TAW; and two wavelength assignment heuristics: first fit and random assignments. Different topologies are included for simulation such as single link, chain, ring, and mesh topology.

The model is composed of simple modules referred to as GMPLS router and compound modules referred to as EndSystem. The GMPLS router module contains all the RWA and reservation algorithms, while the EndSystem module is responsible of generating and analyzing the response to connection request messages.

The EndSystem module composes of Generator and Sink simple modules.

The Generator module generates a certain number of connection requests to randomly chosen destination nodes in the ION.

The Sink module receives the responses to each connection request generated by its counterpart Generator module. The principal task of the Sink module is to analyze the responses and to generate a statistical table.

Note that the Endsystem compound module actually models an end system in an ION. Effectively, the EndSystem module represents one or several end systems such as switches or routers, e.g. such end systems may be an ATM switch or IP routers using MPLS-based protocols. It is also essential to standardize the physical interface between the clients (end systems) and the transport network (ION) which can be designated as the UNI interface. An implementation agreement for the UNI has been proposed by the OIF [30].

Each GMPLS router module is associated to only one Endsystem module. Those modules have the same address. The network model is based on the USA Abilene network that composes of 12 GMPLS router modules scattered at different GigaPoPs; each GMPLS router module is associated to its own Endsystem module. An object-oriented framework has been developed with an UML class diagram (implemented with Rational Rose) of the RWA model which describes the relation and contents of different of classes of the 
simulation framework which are Generator, Sink, GmplsRouter, Node, LinkInfo, NodeInfo, ExplicitRoute and LambdaCap. The details of these classes can b explained on request of readers.

\subsection{Reservation Protocol}

For simplicity, a reservation protocol based on parallel reservation has been implemented in order to test the different routing and wavelength assignment schemes implemented. The reservation scheme and process of messages exchanges are shown in Figure 2 and described as follows:

The Generator sends a "request" message for a new connection to another randomly chosen node. When GMPLS Router receives a "request" message, it calculates an explicit route to the destination requested and assigns a wavelength for this connection and then estimation of the total residual and PMD dispersion, thence broadening time.

If both route and wavelength are available, the GMPLS router reserves in parallel the route and the wavelength. It sends in parallel a "reserve" message to each node of the explicit route, excepted itself. When a node receives the "reserve" message, it checks if the requested wavelength is available on the link to its predecessor in the explicit route. Then it sends a "response" message back to inform the source whether the reservation is successful.

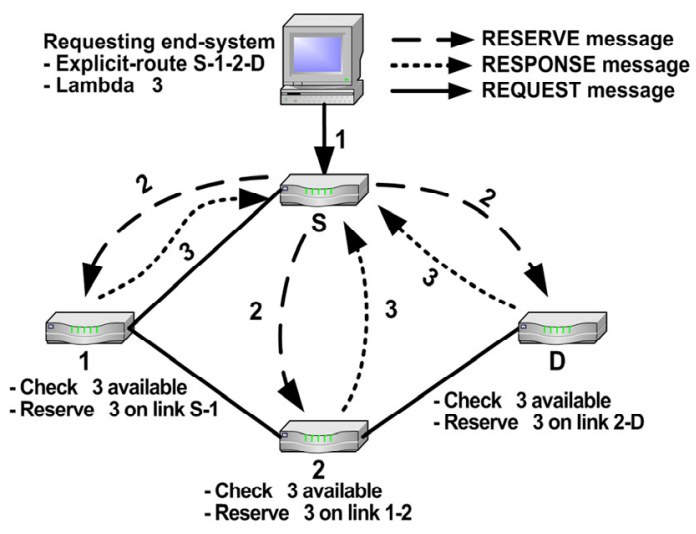

Figure 2. Parallel reservation model

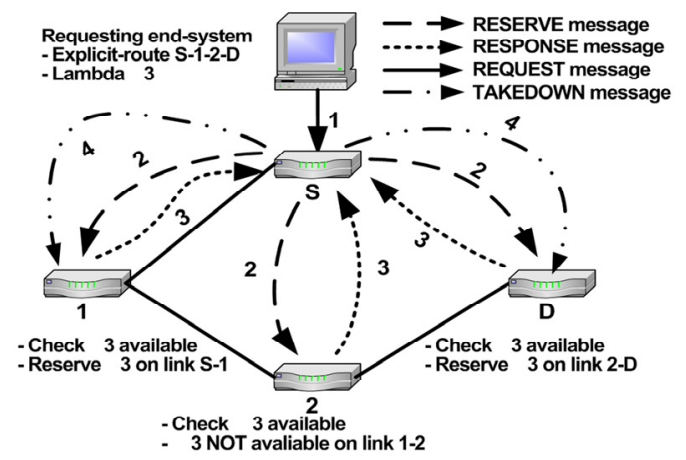

Figure 3. Illustration of the use of TAKEDOWN messages
If the requested wavelength is not available in one or several node on the explicit route, the source will send a TAKEDOWN message to all the nodes, which have already reserved the wavelength on the explicit route to reset it as available (see Figure 3).

\subsection{System Parameters}

The network traffic is generated in terms of connection request, which is a request to establish a lightpath from a source node to a randomly chosen destination node. The connection request arriving at each router is assumed to follow an exponential distribution with mean $\lambda_{\text {poisson }}$ per unit of time. The system variable parameter is $\lambda_{i, j}^{T}$, the total number of wavelengths on each link and the connection requests arrivals. $\lambda_{i, j}^{T}$ changes between 8 and 24 by an increment of 8 . Performance parameters considered are blocking probability $P$ and link utilization $U$.

The blocking probability $P$ is the probability that a connection request is blocked due to no available wavelength for the selected path. The average link utilization $U$ is defined as the percentage of time that all wavelengths of each link in the network are fully utilized, as

$$
U=\frac{\sum_{i, j}^{i<j, i \neq j}\left(1-\frac{\lambda_{i, j}^{a}}{\lambda_{i, j}^{T}}\right)}{\sum_{i, j}^{i<j, i \neq j} \lambda_{i, j}^{T}}
$$

Under the residual dispersion, the constraint for routing is

$$
\sum_{k}^{N_{l}} L_{k, l} \leq L_{D}, L_{P M D}
$$

with the subscript $\mathrm{k}$ indicating fiber path and $N_{l}$ is the total number of the fiber paths that a routing path $l$ is selected. The length limit is set by the dispersion length $L_{D}$ and the PMD effect, $L_{P M D}$, the maximum length due to the PMD effect that enforces a $1 \mathrm{~dB}$ penalty on the eye opening. It is noted that in calculating the total length of the routing route both the linear chromatic and polarization mode dispersion impairments are summed up as they would be superimposed on each other. PMD is a random process and it is modeled with the total average group velocity delay and a Maxwellian distribution. Since the lightpath travels the whole distance from one node to the other, it is reasonable that the average and standard deviation is sufficient for our estimation of the distance and distortion effects.

In addition to the $\mathrm{CD}$ and PMD, nonlinear phase distortion is also critical that would be investigated in a future article. The nonlinear effects [38] include the self phase modulation (SPM), the cross phase modulation $(\mathrm{XPM})$, the four waves mixing (FWM), stimulated 
Raman scattering (SRS) and stimulated Brillouin scattering (SBS). Of these effects SPM and XPM are generated with the change of the phase of the carrier due to the total intensity of the light paths exerted over the core of the fiber and length dependence. The XPM would create a low frequency noise component in the frequency spectra of other lightpaths from lightwave signals of a lightpath. When the utilization is increased the nonlinear effects SPM and XPM are enhanced and thus distortion would affect the quality of the transmitted signals in the lightpaths. Similarly for SRS but the distortion is generated to the lightpaths whose spectra are about $100 \mathrm{~nm}$ away from the signal bands. This would be significant when both $\mathrm{C}$ - and L- bands are employed. These nonlinear effects are not included in this work but will be reported in a future article.

\section{Simulation Results and Discussions}

One of our goals is to estimate the performances of different RWA algorithms by simulations under without and with residual dispersion effects. The blocking probability $P$ versus the average link utilization $U$ is used as the performance criterion. Under the condition that the wavelength conversion is not used, the wavelength continuity constraint would lead to a high level of blocking when the load increases when no dispersion constraints are imposed. We then investigate the performance under the dispersion constraints. Firstly, wavelength assignment schemes are compared. The blocking in the network as a function of the average link utilization is described, analyzed and discussed. Then, various RWA algorithms are tested based on some specific network topologies.

\subsection{Performance under No Constraints of Dispersion Effects}

The first-fit and random wavelength assignment schemes are simulated and presented in this sub-section under non constraints of linear dispersion and nonlinear effects. The blocking probability $P$ is plotted as a function of the link utilization $U$ for different values of $\lambda_{i, j}^{T}$, the total number of wavelengths in a fiber. $\lambda_{i, j}^{T}$ is varied between 8 and 24 by increment of 8 for different schemes, including simple TAW metric, enhanced TAW metric, first-fit and random wavelength assignment are tested. Figure 4 shows the difference of performance between the two wavelength assignments. They represent blocking probability $P$ versus the normalized link utilization for: Figure 4 (a) and (b) $\lambda_{i}^{T}=8 /$ simple TAW, $\lambda_{i, j}^{T}=8 /$ enhanced TAW, (c) and (d) $\lambda_{i, j}^{T}=16 /$ simple TAWW, $\lambda_{i, j}^{T}=16 /$ enhanced TAW, and (e) and (f) $\lambda_{i, j}^{T}=24 /$ simple TAW and $\lambda_{i, j}^{T}=24 /$ enhanced TAW, as indicated.
It is found that the first-fit wavelength assignment performs superior, with much less blocking problem, than the random scheme when the average link utilization is between $40 \%$ and $60 \%$, and when the link utilization becomes relatively high, the difference between these two wavelength assignments is very small. The results are independent with either the TAW type or the number of total wavelengths.

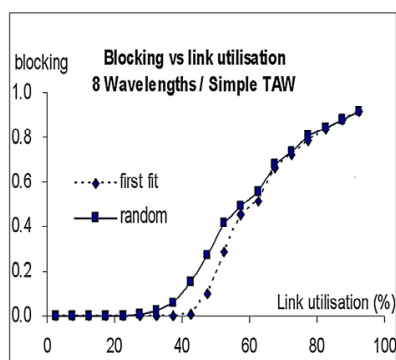

(a)

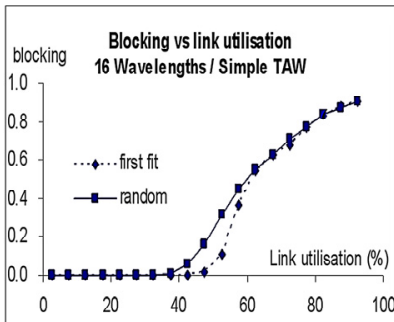

(c)

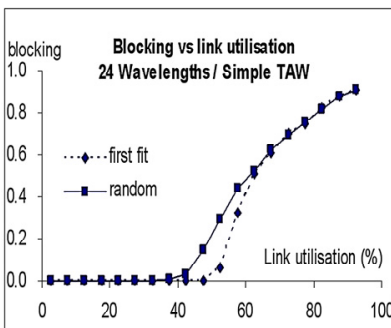

(e)

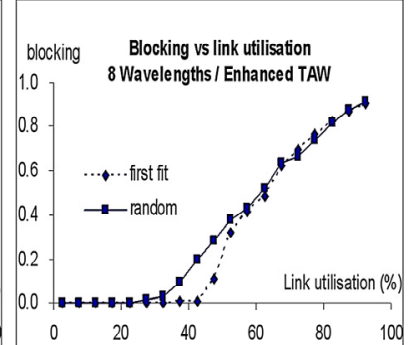

(b)

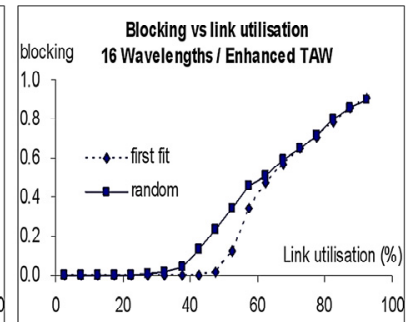

(d)

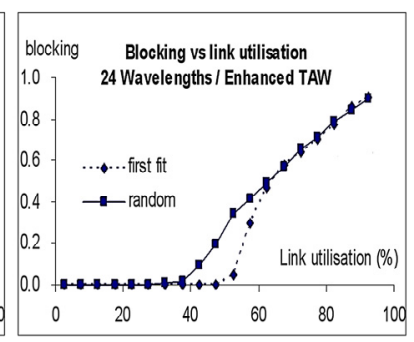

(f)
Figure 4. Blocking probability versus link utilization with total number of wavelength for First-fit and random wavelength assignments - no linear dispersion and nonlinear effects.

Shown in Figure 5 is the difference of performance between two metrics used simple and enhanced TAW. When the link utilization is relatively low, the difference is not much, but with link utilization becomes high, enhanced TAW performs better. In general, it has been observed that the enhanced TAW metric performs better than the simple TAW metric at higher utilization. One reason is that the enhanced metric tries to minimize the weight of the explicit route while still trying to minimize the probability of blocking for further requests. Conversely, the simple TAW metric does not take into account any probabilities. It just tries to minimize the total link utilization on a possible explicit route from the 
ingress node to the egress node.

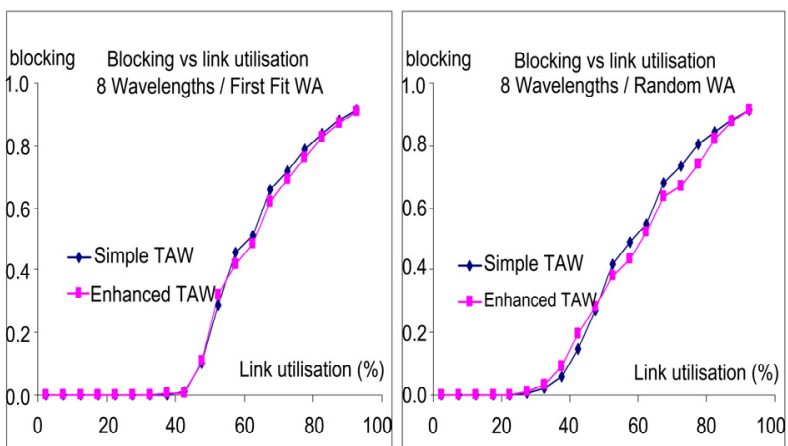

(a)

(b)

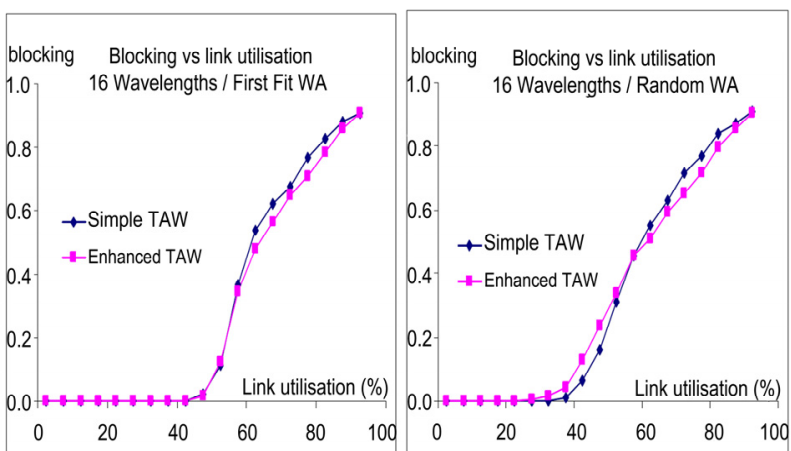

(c)

(d)

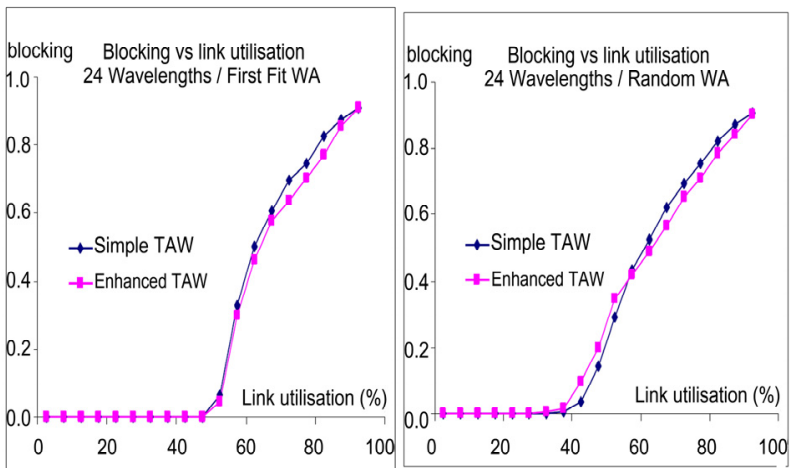

(e)

(f)

Figure 5. Blocking probability versus link utilization with total number of wavelength for simple and enhanced TAW.

\subsection{Performance under Constraints of Dispersion Effects for Specific Networks}

The purpose of this section is to test the affection of network topologies on different RWA algorithms under the constraints of linear dispersion and nonlinear effects. Specific networks of 5 node ring and a specific long haul backbone mesh network (see Figure 6) are chosen so that the lengths of the fiber physical paths can be determined.

Now let

$$
\begin{gathered}
\mathrm{A}=\left\{\mathrm{A}_{1} \div \mathrm{A}_{4}\right\}=\{4 \text {-node chain, 8-node chain, 5-node } \\
\text { ring, Specific Net }\}
\end{gathered}
$$

be the set of four topologies (Networks with assigned codes of A2, A3 and A4) to be investigated including a 4-nodes (A1) and 8-nodes chains (A2), a 5-nodes ring (A3), and the Specific Net (Figure 6 as A4), respectively. A total wavelengths number of 24 which is chosen with $50 \mathrm{GHz}$ channel spacing for the study of the traffic performance o the networks under no dispersion effects and with linear CD and PMD effects. Note that the dispersion due to nonlinear self phase effect is not considered as a constraint [40]. The network routing is subjected to the linear dispersion constraints given in eq.(6) and (3) and (4). For the same reason as mentioned above, we compare two wavelength assignment heuristics - the first-fit and random wavelength assignments.

The simulation results are shown in Fiugre 8 and we can make the following observations. Firstly, under the case of the simplest topology - single link, suppose there are $n$ total wavelengths on the link, then when the number of requests is smaller than $n$, the block will never happen. This is because there must be unused wavelength(s) on the link, and no matter which wavelength assignment heuristic is adopted, the source chooses the wavelength from those unused. Secondly, when the link utilization is low, there is no difference between First-fit and random wavelength assignments, since the connection requests can always be satisfied. When link utilization becomes higher, the block happens. In the same topology, the First-fit WA has a lower blocking probability than random wavelength assignment. But when link utilization is very high, the difference between these two wavelength assignments becomes narrower.

It also can be seen that the difference between first-fit and random wavelength assignment heuristics of the 4nodes chain is quite small, but that of 8-nodes chain becomes larger. Naturally the reason is destinations of connections are chosen randomly, so with 8-nodes chain there should be more multi-hop connection requests than that with 4-nodes chain. The first-fit heuristic tends to pack all of the in-use wavelengths toward the lower end of the wavelength space so that more wavelengths with higher indexes can be used for longer-hop connections. Thus on 8-nodes chain the advantage of First-fit heuristic becomes more obvious.

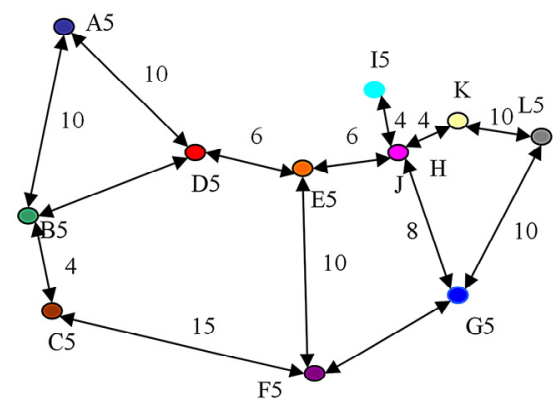

Figure 6. Specific Net with Cities and Distance (in number of spans). A typical long haul back bone optically amplified 
dispersion managed network - the number indicates the number of spans in multiples of $100 \mathrm{~km}$ spans with dispersion compensating module and optical amplifiers. Capital letters indicate the sites for IP routers and OXCs. Residual dispersion is taken as $2 \%$ of the link distance.

The ring and 4-node chain topologies give much lower blocking probability as compared with 8-node chain and mesh topologies (The Specific Net of Figure $6)$.

We now demonstrate the two link metrics: simple TAW and enhanced TAW. Link metrics are used in path selection. In the chain topologies, there is only one path between the source and the destination, so there is no difference between simple TAW and enhanced TAW. The blocking probability for a 5-nodes ring (A3) and Specific Net (A4) topologies with a total of 24 wavelength channels. A3 network is set under the condition of equal number of spans per link of 5 between nodes with $100 \mathrm{~km}$ completely dispersion compensated per span - that is a total of $500 \mathrm{~km}$ of dispersion compensated fibers and optical amplifiers. A residual dispersion is set at $2 \%$ of the dispersion of the uncompensated dispersion of the lowest dispersion value (e.g. $2 \%$ of $17 \mathrm{ps} / \mathrm{nm} / \mathrm{km}$ of standard single mode optical fiber). An average PMD first order value of 0.1 $\mathrm{ps} /(\mathrm{km})^{1 / 2}$ is also included. As can be seen from Figure 7, the enhanced TAW performs better than simple TAW, because the enhanced TAW takes into consideration the further requests and tries to minimize the probability of blocking for future, while simple TAW only choose the link with a light load. The effects of CD and PMD have shown clearly an increase in the blocking probability when the utilization of the link paths is increased. This is expected due to the limitation of the dispersion budget given in (2) and (3). We note here that no statistical property of the PMD is taken into account and that we may expect some sudden fluctuation of the blocking probability in practice.

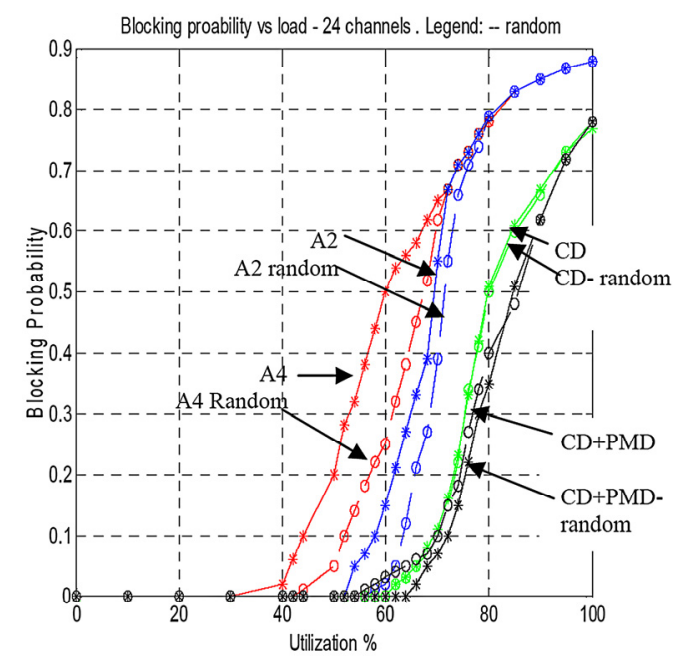

Figure 7. Blocking probability versus link utilization with total number of wavelength is 24 for 2 kinds of topology under with and without constraints of linear chromatic dispersion (CD bold circle dotted line) and CD + PMD (random) effects (square dotted line). Legend: dotted line indicates "random".

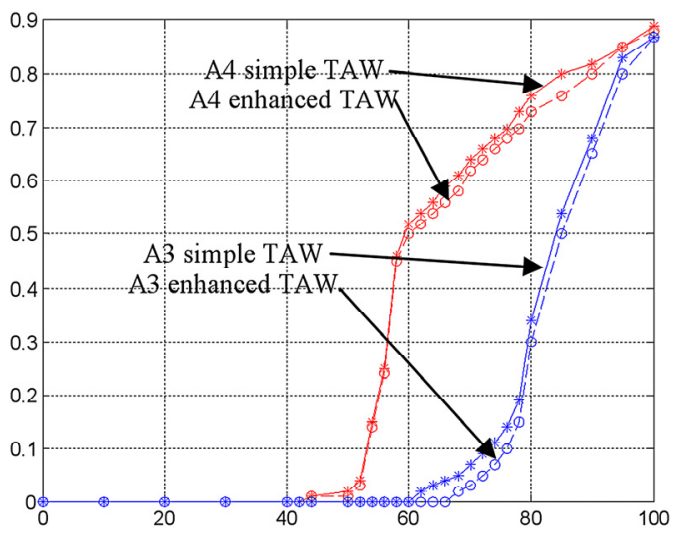

(a)

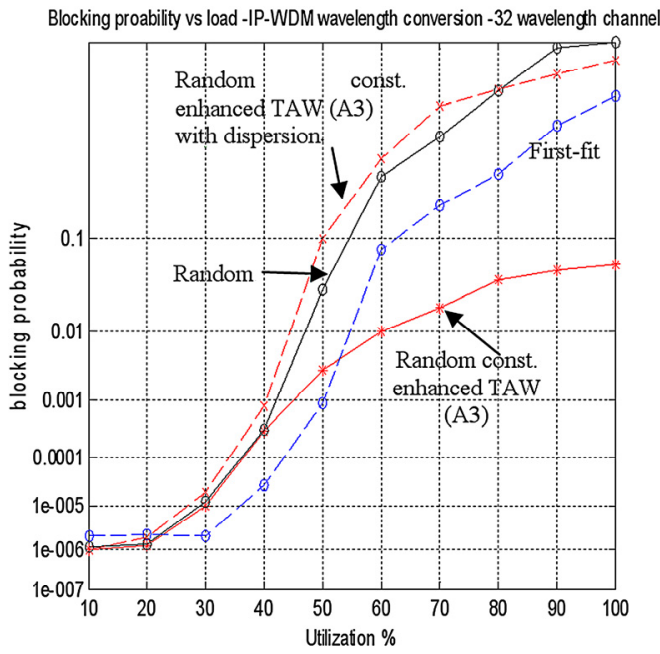

(b)

Figure 8. Simple TAW vs. Enhanced TAW on node-ring and sample network (Figure 6) (a) without (b) with dispersion constraints using random and first-fit strategy.

Also, numerous simulations have been conducted to test these RWA heuristics on different traffic schemes and total available wavelengths. The traffic schemes include uniform, exponential and Poisson distribution, and the number of total available wavelengths is various among 8,16 and 24 . There is no obvious impact on the blocking probability due to different traffic schemes and the number of total wavelengths. The first fit WA performs better than random WA, and enhanced TAW performs better than simple TAW. Figure 8(a) and (b) shows a contrast of the blocking probability of the simple TAW and enhanced TAW under without and with dispersion constraints with different algorithms.

Concerning with TAKEDOWN message of reservation scheme, in our previous simulations, the 
TAKEDOWN message never appears. This is because when a wavelength is reserved on some link(s), the UPDATE messages will be sent to every node in the network. When a source calculates the explicit routes, it requires the knowledge of whether a wavelength is unused or not, so it would not seek other nodes to reserve those unavailable ones. There is one case that the TAKEDOWN message would be used: after a wavelength is reserved on some link, and before the UPDATE message reaches a source, this source receives a connection request and estimates some explicit route using that wavelength on that link. Then the source seeks for the downstream node of that link to reserve the wavelength, which is already reserved for some other connection. Clearly, the node responds negatively, and TAKEDOWN messages are to be sent from the source to other nodes on that explicit route.

\section{Concluding Remarks}

In this paper we have presented a simulation model on GMPLS-based all-optical networks using OMNeT++ to study the traffic performance of RWA of channels within the C-band of optical fibers under the influences of the dispersion effects due to chromatic and polarization mode dispersion. The blocking probabilities of two types of wavelength assignment heuristics and two types of link metrics are investigated.

It has been found that when the link utilization is low, there is no difference in blocking probability between first fit and random wavelength assignments. Almost all the connection requests can be satisfied. When link utilization becomes high, first fit performs better than random wavelength assignment. However, when link utilization is close to $100 \%$, the difference between the two wavelength assignment heuristics becomes small again. At higher link utilizations, blocking increases exponentially when link utilization increases.

For the link metrics, we find, in a practical network such as a ring or a mesh network, the enhanced TAW link metric has a lower blocking probability than simple TAW. Further the enhanced TAW metric performs better at high link utilizations than the simple TAW metric particularly when the number of wavelengths per fiber is low. Conversely, at low link utilization, both metrics have very similar results whatever the number of wavelengths are used. Moreover, we also design a contention case to illustrate the use of TAKEDOWN message. Under this situation, the random wavelength assignment performs better than first fit wavelength assignment by spreading the chosen wavelengths.

In the simulation we only examine two simplest wavelength assignment heuristics: first-fit and random wavelength assignment schemes. They yield similar performance when associated with any routing and reservation schemes implemented in this work. We believe more heuristics should be tested and compared.
Also, the wavelength convention (both full-convention and part-convention) can be considered. For the contention case, we wonder some improvements can be done on the first fit wavelength assignment heuristic, to make its blocking probability not worse than random heuristic.

The effects of chromatic dispersion and PMD are also included as constraints of the routing and wavelength assignment. These effects are significant for $10 \mathrm{~Gb} / \mathrm{s}$ optical networks. The random scheme may not offer the best due to the effects of the residual dispersion. So the first-fit would offer better performance under these dispersion constraints. Nonlinear dispersion effects in RWA in optical networks such as self phase modulation will be included in future works. These effects are critical when the number of wavelength channels are increased, thus the total average power and intensity imposed on the fiber guiding region. This is very critical for network management in practice as the symbol rates are increased. The $40 \mathrm{~Gb} / \mathrm{s}$ is emerging as the rate of next generation networks and the linear dispersion and nonlinear phase distortion are even much more critical with much lower tolerance for distortion and must be taken into account in the RAW management strategy. This will be reported in a future article. The randomness and statistical property of the PMD is included only with its mean value. This clearly allows the expected traffic performance and not to the details of the blocking that may happen higher or lower from time to time.

We have also assumed that the lightwaves of the lightpaths are amplitude modulated with either NRZ or $\mathrm{RZ}$ formats. It is more likely that the RZ format is used as it is the preferred format. Other modulation formats such as different phase shift keying [39], continuous phase shift keying or frequency shift keying have also been intensively investigated. They would be taken as the critical feature to combat impairments in long haul optical DWDM networks and RWA management strategy.

\section{Acknowledgement}

The author acknowledges the OMNeT++ programming assistance from C. Cieutat.

\section{References}

[1] IEEE Workshop on Advanced Modulation Formats, San Francisco, USA, 2004.

[2] N.Y. Ken-ichi Sato, et al., "GMPLS-Based Photonic Multilayer Router (Hikari Router) Architecture: An Overview of Traffic Engineering and Signaling Technology," IEEE Communications, vol. 40, no. 3, pp. 96-101, March 2002.

[3] G.N. Rouskas and H.G. Perros, "A Tutorial on Optical Networks," Networking 2002 Tutorials, Lecture Notes in Computer Science, vol. 2497, 2002, pp. 155-193. 
[4] R. Ramaswami and K.N. Sivarajan, "Optical Networks: A Practical Perspective," 2nd Edition, Morgan Kaufmann, 2002.

[5] P.A. Smith, et al., "Generalized Multi-Protocol Label Switching (GMPLS) Architecture," draft-ietf-ccampgmpls-architecture-02.txt, 2002.

[6] K. Kompella, et al., "Routing Extensions in Support of Generalized MPLS," draft-ietf-ccamp-gmpls-routing04.txt, 2002.

[7] D. Katz, D. Yeung, and K. Kompella, "Traffic engineering extensions to OSPF," draft-katz-yeung-ospftraffic-10.txt, December 2003.

[8] H. Smith and T. Li, "IS-IS Extensions for Traffic Engineering," draft-ietf-isis-traffic-05.txt, August 2003.

[9] L. Berger, et al., "Generalized MPLS - Signaling Functional Description," draft-ietf-mpls-generalizedsignaling-08.txt, 2002.

[10] L. Berger, et al., "Generalized MPLS Signaling RSVP-TE Extensions," draft-ietf-mpls-generalized-rsvpte-07.txt, 2002

[11] B. Jamoussi, ed., et al., "Constraint-Based LSP Setup using LDP," RFC 3212, IETF Standards Track.

[12] J. Varga, OMNeT++, 2003. http://whale.hit.bme.hu/omnetpp/.

[13] D. Banerjee and B. Mukherjee, "A Practical Approach for Routing and Wavelength Assignment in Large Wavelength-Routed Optical Networks," IEEE Journal Selected Areas in Communications, vol. 14, no. 5, pp. 903-908, June 1996

[14] H. Zang, J.P. Jue, and B. Mukherjee, "A Review of Routing and Wavelength Assignment Approaches for Wavelength-Routed Optical WDM Networks," Optical Networks, vol. 1, no. 1, pp. 47-60, January 2000.

[15] Apostolopoulos, et al., "QoS Routing Mechanisms and OSPF Extensions," RFC 2676, 1999.

[16] R. Dutta and G.N. Rouskas, "A Survey of Virtual Topology Design Algorithms for Wavelength Routed Optical Networks," Optical Networks, vol. 1, no. 1, pp. 73-89, January 2000.

[17] G. Xiao and Y. Leung, "Algorithms for Allocating Wavelength Converters in All-Optical Networks," IEEE/ACM Transactions on Networking, vol. 7, no. 4, pp. 545-557, August 1999.

[18] E. Leonardi, M. Mellia, and M.A. Marsan, "Algorithms for the Logical Topology Design in WDM All-Optical Networks," Optical Networks, vol. 1, no. 1, pp.35-46, January 2000.

[19] Y. Zhang, et al., "An Efficient Heuristic for Routing and Wavelength Assignment in Optical WDM Networks," IEEE ICC 2002, vol. 5, pp. 2734-2739, May 2002.

[20] A. Birman, "Computing Approximate Blocking Probabilities for a Class of All-Optical Networks," IEEE Journal Selected Areas in Communications, vol. 14, no. 5, pp. 852-857, June 1996.

[21] R. Ramaswami and K.N. Sivarajan, "Design of Logical Topologies for Wavelength Routed Optical Networks," IEEE Journal Selected Areas in Communications, vol. 14, no. 5, pp. 840-851, June 1996.
[22] O. Gerstel and S. Kutten, "Dynamic Wavelength Allocation in All-Optical Ring Networks," IEEE ICC'97, vol. 1, pp. 432-436, June 1997.

[23] J. Moy, OSPF Version 2, RFC 2328, 1998.

[24] T.F. Asztalos, N.M. Bhide, and K.M. Sivalingam, "Adaptive Weight Functions for Shortest Path Routing Algorithms for Multi-Wavelength Optical WDM Networks," IEEE ICC'2000, New Orleans, LA, pp. 1330-1334, June 2000.

[25] O. Gerstel, "On The Future of Wavelength Routing Networks," IEEE Network, vol. 10, no. 6, pp. 14-20, November-December 1996.

[26] P.H. Ho and H.T. Mouftah, "Path Selection with Tunnel Allocation in the Optical Internet Based on Generalized MPLS Architecture," IEEE ICC 2002, vol. 5, pp. 26972701, May 2002.

[27] J. Zheng and H.T. Mouftah, "Routing and Wavelength Assignment for Advance Reservation in WavelengthRouted WDM Optical Networks," IEEE ICC 2002, vol. 5, pp. 2722-2726, May 2002.

[28] R.S. Ramaswami, "A. Distributed network control for wavelength routed optical networks," IEEE Infocom, San Francisco, CA, USA, pp. 138-147, March 1996.

[29] R. Ramaswami and K.N. Sivarajan, "Routing and Wavelength Assignment in All-Optical Networks," IEEE/ACM Transactions on Networking, vol. 3, no. 5, pp. 489-500, October 1995.

[30] Working Group: Architecture, O.P., PLL, \& Signaling Working Groups, User Network Interface (UNI) 1.0.

[31] Signaling Specification, 2001, Optical Internetworking Forum (OIF).

[32] A. Birman and A. Kershenbaum, "Routing and Wavelength Assignment Methods in Single-Hop AllOptical Networks with Blocking," IEEE Infocom'95, vol. 2, pp. 431-438, April 1995.

[33] B. Mukherjee, et al., "Some Principles for Designing a Wide-Area WDM Optical Network," IEEE/ACM Transactions on Networking, vol. 4, no. 5, pp. 684-695, October 1996.

[34] I. Widjaja and A.I. Elwalid, "Study of GMPLS Lightpath Setup over Lambda-Router Networks," IEEE ICC 2002, vol. 5, pp. 2707-2711, May 2002.

[35] D. Banerjee and B. Mukherjee, "Wavelength-Routed Optical Networks: Linear Formulation, Resource Budgeting Tradeoffs, and Reconfiguration Study," IEEE/ACM Transactions on Networking, vol. 8, no. 5, pp. 598-607, October 2000.

[36] X. Zhang and C. Qiao, "Wavelength Assignment for Dynamic Traffic in Multi-fiber WDM Networks," IEEE ICC'98, pp. 479-485, October 1998.

[37] G.P. Agrawal, "Fiber-optic communications systems," 3rd Edition, John Wiley \& Sons, 2001.

[38] I. Kaminov and T. Koch, "Optical Fiber Communications," vol. 3.1, Academic Press.

[39] C. Wree, "Differential phase shift keying for long haul fiber optic transmission based on direct detection," Doctoral Thesis Dissertation, CA University of Kiel, 2002. 